\title{
Global Executive Composite
}

National Cancer Institute

\section{Source}

National Cancer Institute. Global Executive Composite. NCI Thesaurus. Code 121474.

An overall index of the Behavior Rating Inventory of Executive Function which takes into account all of the clinical scales and represents the individual's overall executive function. 